\title{
Simplifying additivity problems using direct sum constructions
}

\author{
Motohisa Fukuda ${ }^{1}$, Michael M. Wolf ${ }^{2}$ \\ ${ }^{1}$ Statistical Laboratory, Centre for Mathematical Sciences, University of Cambridge \\ ${ }^{2}$ Max-Planck-Institute for Quantum Optics, Hans-Kopfermann-Str. 1, D-85748 Garching, Germany.
}

(Dated: August 20, 2021)

\begin{abstract}
We study the additivity problems for the classical capacity of quantum channels, the minimal output entropy and its convex closure. We show for each of them that additivity for arbitrary pairs of channels holds iff it holds for arbitrary equal pairs, which in turn can be taken to be unital. In a similar sense, weak additivity is shown to imply strong additivity for any convex entanglement monotone. The implications are obtained by considering direct sums of channels (or states) for which we show how to obtain several information theoretic quantities from their values on the summands. This provides a simple and general tool for lifting additivity results.
\end{abstract}

\section{INTRODUCTION}

A central question in classical and quantum information theory is, how much information can be transmitted through a given noisy channel. For classical channels the maximal asymptotically achievable rate - the capacity — was derived in the seminal work of Shannon [1]. For quantum channels, however, the matter is complicated by the existence of entanglement and the possibility of exploiting it in the encoding to protect information against decoherence. If one excludes this possibility, a capacity formula for the transmission of classical information through quantum channels was proven by Holevo [2] and Schumacher and Westmoreland [3] (HSW). Since then, considerable effort was devoted to the question whether (or in which cases) entangled inputs can lead to rates beyond the HSW capacity. This issue-the additivity problem for the HSW capacity-is still undecided, although for several classes of channels additivity has been shown to be true, i.e., entanglement does not seem to help in any case (see, e.g., [4, 5, 6, 7] and references therein). Instead, other additivity problems appeared which are similar in spirit but concern very different quantities like the minimal output entropy and the entanglement of formation, an entanglement measure for bipartite states for which in addition strong super-additivity has been conjectured.

A major conceptional insight was then gained in [8, 9, 10, 11] where it was shown that all these additivity problems are globally equivalent in the sense that if additivity holds for one of these quantities in general, then it does so for all of them. Here 'in general' means that it has to be true for arbitrary pairs of channels (or states), a condition we will call strong additivity.

In this work we present a further conceptional simplification of these and related additivity problems. We show that strong additivity is implied by weak additivity, meaning additivity for arbitrary pairs of equal channels or states. Moreover, based on [12] we argue that it suffices to consider pairs of identical unital channels only. This observation may be a small step on a notorious path but it might guide future research as it for instance underlines recent attempts to understand the asymptotic structure of tensor powers of unital channels [13]. Moreover, one may think of other additivity questions than the ones stated above for which our techniques could be of use. In particular, we think of regularized quanti- ties (like quantum capacities (cf. [14, 15]) or certain entanglement measures) for which weak additivity holds by definition.

Our main tool is the use of direct sums of channels or states. For the latter case similar constructions appeared in [16, 17, 18]. We begin with a discussion of direct sum channels. This will contain more than what is needed for the subsequent additivity results as we think that these tools might be of independent interest.

\section{DIRECT SUMS OF QUANTUM CHANNELS}

We consider direct sums of channels, i.e., completely positive and trace preserving maps of the form $T=\oplus_{i} T_{i}$, where each $T_{i}$ is a channel in its own right. Our aim in this section is to express information theoretic functionals of $T$ in terms of their values for the $T_{i}$ 's. The definition of the quantities appearing in the following proposition will be given in the proof.

Proposition 1 (Direct sums) Consider a direct sum $T=$ $\oplus_{i=1}^{n} T_{i}, n \in \mathbb{N}$ of arbitrary finite dimensional channels. Then

1. Minimal output $\alpha$-Renyi entropy $(\alpha \geq 1)$ :

$$
S_{\min , \alpha}(T)=\min _{i}\left[S_{\min , \alpha}\left(T_{i}\right)\right]
$$

2. Coherent information:

$$
J(T)=\max _{i}\left[J\left(T_{i}\right)\right],
$$

3. Mutual information:

$$
\begin{aligned}
I(T) & =\max _{\left\{\lambda_{i}\right\}} S\left(\left\{\lambda_{i}\right\}\right)+\sum_{i} \lambda_{i} I\left(T_{i}\right), \\
& =\log \sum_{i} 2^{I\left(T_{i}\right)},
\end{aligned}
$$

where $\left\{\lambda_{i}\right\}$ is a probability distribution and $S\left(\left\{\lambda_{i}\right\}\right)$ its entropy.

4. HSW capacity:

$$
\begin{aligned}
\chi(T) & =\max _{\left\{\lambda_{i}\right\}} S\left(\left\{\lambda_{i}\right\}\right)+\sum_{i} \lambda_{i} \chi\left(T_{i}\right) \\
& =\log \sum_{i} 2^{\chi\left(T_{i}\right)} .
\end{aligned}
$$


Remark: Let us briefly comment on the interpretation of the above formulas. Concerning the HSW capacity, classical information can either be sent through the channels $T_{i}$ or it can be encoded in the choice of blocks $i=1, \ldots, n$. Eq.(5) shows exactly the competition between these two ways of communicating classical information. For the quantum mutual information, which gives the entanglement assisted capacity [19], we obtain the same interpretation (note that the '2' comes from the fact that we take log in base 2). The coherent information is related (via regularization) to the quantum capacity [20]. In this case encoding information in the choice of blocks is not possible - this would be purely classical as all the coherences get lost. Similarly, for the minimal output entropies the minimum is obtained by putting all the weight into the least noisy channel.

Proof. 1. The $\alpha$-Renyi entropy is defined as

$$
S_{\alpha}(\rho)=\frac{1}{1-\alpha} \log \operatorname{tr}\left[\rho^{\alpha}\right]=\frac{\alpha}{1-\alpha} \log \|\rho\|_{\alpha},
$$

for $0 \leq \alpha \leq \infty$. Here $\|\cdot\|_{p}$ is the Schatten $p$-norm. When $\alpha=1$ the functional is defined by its limit which is the minimal output entropy $S_{\min }(T)=\inf _{\rho} S(T(\rho))$ with $S(\rho)=-\operatorname{tr} \rho \log \rho$ the von Neumann entropy. Let us consider this case first.

As the direct sum $\oplus_{i} T_{i}$ erases the off-diagonal blocks so that all possible outputs can be obtained upon block-diagonal inputs we can restrict to $\rho=\oplus_{i} \tilde{\rho}_{i}$. Here $\tilde{\rho}_{i}$ is not necessarily normalized so that the weights $\operatorname{tr}\left[\tilde{\rho}_{i}\right]=: \lambda_{i}$ form a probability distribution. Writing $\rho_{i}:=\tilde{\rho}_{i} / \lambda_{i}$ and using the concavity of von Neumann entropy we get

$$
S\left(\oplus_{i} T_{i}\left(\lambda_{i} \rho_{i}\right)\right) \geq \sum_{i} \lambda_{i} S\left(T_{i}\left(\rho_{i}\right)\right) .
$$

This leads to Eq. (1) when $\alpha=1$. For $\alpha>1$ the minimization of $S_{\alpha}(T(\rho))$ amounts to a maximization of $\|T(\rho)\|_{\alpha}$ and the result follows from convexity of $\|\cdot\|_{\alpha}$ in a similar way.

2. The coherent information is defined as

$$
J(T)=\sup _{\rho} S(T(\rho))-S(T \otimes \operatorname{id}(\Psi)),
$$

where $\Psi$ is a purification of $\rho$ such that $\rho=\operatorname{tr}_{B} \Psi$. Since $T$ and $T \otimes$ id erase the off-diagonal blocks we can replace $\rho$ and $\Psi$ by their diagonal blocks: $\oplus_{i} \lambda_{i} \rho_{i}$ and $\oplus_{i} \lambda_{i} \Psi_{i}$. Here, $\Psi_{i}$ is an extension of $\rho_{i}$. Since the conditional entropy $S\left(\rho_{A B}\right)-S\left(\rho_{A}\right)$ is concave in $\rho_{A B}$ [21] considering a convex decomposition of each $\Psi_{i}$ into pure states shows Eq.(2) in a similar way as above.

\section{The mutual information defined as}

$$
I(T)=\sup _{\rho} S(\rho)+S(T(\rho))-S(T \otimes \mathrm{id}(\Psi))
$$

is concave in $\rho$ so the maximum will be achieved by a block diagonal $\rho=\oplus_{i} \lambda_{i} \rho_{i}$ for $T=\oplus_{i} T_{i}$. To see this, let $V=$ $\oplus_{j} \exp \{2 \pi \mathrm{i} j / n\} I_{j}$ and average $V^{k} \rho V^{* k}$ over $k=1, \ldots, n$. Take a purification $\Psi$ of $\rho=\oplus_{i} \lambda_{i} \rho_{i}$, and then replace $\Psi$ by its diagonal blocks: $\oplus_{i} \lambda_{i} \Psi_{i}$ as before. However, each $\Psi_{i}$ is a purification of $\rho_{i}$ in this case. Indeed, suppose $\rho_{i}=$ $\sum_{j} p_{i j}|i j\rangle\langle i j|$, where $\{|i j\rangle\}_{j}$ is an orthonormal basis in the $i$ th subspace. Then, $\Psi$ is

$$
\sum_{i j k l} \sqrt{\lambda_{i} \lambda_{k} p_{i j} p_{k l}}|i j\rangle\langle k l|\otimes| i j\rangle\langle k l|,
$$

and its $i$ th diagonal block $\lambda_{i} \Psi_{i}$ is

$$
\lambda_{i} \sum_{j l} \sqrt{p_{i j} p_{i l}}|i j\rangle\langle i l|\otimes| i j\rangle\left\langle i l\left|=\lambda_{i}\right| \Psi_{i}\right\rangle\left\langle\Psi_{i}\right| .
$$

Here, $\left|\Psi_{i}\right\rangle=\sum_{j} \sqrt{p_{i j}}|i j\rangle \otimes|i j\rangle$. Exploiting this together with $S(\lambda \rho)=\lambda(S(\rho)-\log \lambda)$ then gives Eq.(3). Eq.(4) follows then from determining the optimal $\lambda_{i}$ via Lagrange multipliers in the following way. The maximization problem of $S\left(\left\{\lambda_{i}\right\}\right)+\sum \lambda_{i} c_{i}$ for a probability distribution $\left\{\lambda_{i}\right\}$ amounts then to maximizing

$$
S\left(\left\{\lambda_{i}\right\}\right)+\sum_{i} \lambda_{i} c_{i}+\Lambda\left(\sum \lambda_{i}-1\right),
$$

where $\Lambda$ is the Lagrange multiplier. Taking partial derivatives we obtain for extremal $\left\{\tilde{\lambda}_{i}\right\}$ :

$$
\begin{aligned}
-\log \tilde{\lambda}_{i}-\frac{1}{\ln 2}+c_{i}+\Lambda & =0 \quad \forall i \\
\sum_{i} \tilde{\lambda}_{i}-1 & =0
\end{aligned}
$$

Hence (12) shows $c_{i}-\log \tilde{\lambda}_{i}$ is a constant, say, $C$ for $\forall i$, and by 13 we get $C=\log \sum_{i} 2^{c_{i}}$. Therefore

$$
S\left(\left\{\tilde{\lambda}_{i}\right\}\right)+\sum_{i} \tilde{\lambda}_{i} c_{i}=\sum_{i} \tilde{\lambda}_{i} C=\log \sum_{i} 2^{c_{i}} .
$$

As this is lower bounded by $\min _{i}\left[I\left(T_{i}\right)\right]$ it must be the maximum.

4. The HSW capacity $\chi$ is given by

$$
\begin{aligned}
& \chi(T)=\sup _{\rho} S(T(\rho))-H_{T}(\rho) \\
& \text { where } \quad H_{T}(\rho)=\inf _{\sum p_{k} \rho_{k}=\rho} \sum_{k} p_{k} S\left(T\left(\rho_{k}\right)\right) .
\end{aligned}
$$

Here, $H_{T}(\rho)$ is the convex closure of the output entropy; $\left\{p_{k}\right\}$ is a probability distribution and $\rho_{k}$ are density matrices. The r.h.s. of Eq. 15) for a fixed average input state $\rho$ is a constraint $H S W$ capacity which we will denote by $\chi(T, \rho)$. Since the inputs can again be assumed to be block-diagonal we have:

$$
\begin{aligned}
\chi\left(\oplus_{i} T_{i}, \oplus_{i} \lambda_{i} \rho_{i}\right) & =S\left(\oplus_{i} \lambda_{i} T_{i}\left(\rho_{i}\right)\right)-\sum_{i} \lambda_{i} H_{T_{i}}\left(\rho_{i}\right) \\
& =S\left(\left\{\lambda_{i}\right\}\right)+\sum_{i} \lambda_{i} \chi\left(T_{i}, \rho_{i}\right)
\end{aligned}
$$

The first equality is explained by the fact that since the von Neumann entropy is concave there is an optimal decomposition of $\oplus_{i} \lambda_{i} \rho_{i}$ for which each state has its support in one of the diagonal blocks. The second equality comes from $S\left(\lambda_{i} \rho_{i}\right)=\lambda_{i}\left(S\left(\rho_{i}\right)-\log \lambda_{i}\right)$. Taking the supremum over all states $\left\{\rho_{i}\right\}$ then leads to Eq. (5). Again, Eq. (6) is obtained by using Lagrange multipliers as above. In fact, for unital channels (6) has been obtained in [30]. 


\section{SIMPLIFYING ADDITIVITY PROBLEMS}

Let us now turn to the additivity conjectures and exploit Prop 1 in order to show that in several cases weak additivity (for equal channels or states) implies strong additivity (i.e., for different ones).

Proposition 2 (Reduction for channels) The following (in-) equalities hold for arbitrary pairs of different channels $T_{1}$ and $T_{2}$ iff they hold for arbitrary equal pairs $T_{1}=T_{2}$.

1. $S_{\min , \alpha}\left(T_{1} \otimes T_{2}\right)=S_{\min , \alpha}\left(T_{1}\right)+S_{\min , \alpha}\left(T_{2}\right)$, for any $\alpha \geq 1$.

2. $\chi\left(T_{1} \otimes T_{2}\right)=\chi\left(T_{1}\right)+\chi\left(T_{2}\right)$.

3. $H_{T_{1} \otimes T_{2}}(\rho) \geq H_{T_{1}}\left(\rho_{1}\right)+H_{T_{2}}\left(\rho_{2}\right)$ for all states $\rho$ with respective subsystems $\rho_{1}, \rho_{2}$.

4. $H_{T_{1} \otimes T_{2}}\left(\rho_{1} \otimes \rho_{2}\right)=H_{T_{1}}\left(\rho_{1}\right)+H_{T_{2}}\left(\rho_{2}\right)$ for all product states $\rho_{1} \otimes \rho_{2}$.

Remark: The conjectured equality in 1 . is the additivity of the minimal output entropy when $\alpha=1$ [22], and it becomes the multiplicativity of maximal output $p$-norms for $p=\alpha>1$. This was conjectured to be true for all $\alpha \in[1,+\infty]$ before a counterexample was found [23] ruling out all values $\alpha>4.79$. The equation in 2. is the conjectured additivity of the HSW capacity, which gives the classical capacity as long as entangled states are not allowed to be used in the encoding [2, 3]. The additivity would show that the HSW capacity itself is the unconstrained classical capacity of quantum channels. The conjectures 3. and 4. are called strong superadditivity and additivity of the convex closure of the output entropy. When $T_{1}, T_{2}$ are partial traces they become strong superadditivity and additivity of entanglement of formation, respectively, which we discuss in greater detail below.

We note that Prop 2 remains valid in the case where 'arbitrary channels' refers to a restricted set of channels which is closed under direct sums and tensor products.

Proof. 1. Let $\sigma_{1}$ and $\sigma_{2}$ be optimal output states for $T_{1}$ and $T_{2}$ respectively. Then, form the following two channels:

$$
T_{1}^{\prime}(\rho)=T_{1}(\rho) \otimes \sigma_{2}, \quad T_{2}^{\prime}(\rho)=\sigma_{1} \otimes T_{2}(\rho) .
$$

It is not difficult to see that $T_{1} \otimes T_{2}$ and $T_{1}^{\prime} \otimes T_{2}^{\prime}$ share the additivity property. Hence we can assume that $T_{1}$ and $T_{2}$ have the same optimal output: $S_{\min , \alpha}\left(T_{1}\right)=S_{\min , \alpha}\left(T_{2}\right)$. If we apply first weak additivity and then Prop. 1.1. we obtain:

$$
\begin{aligned}
& S_{\min , \alpha}\left(\left(\left(T_{1} \oplus T_{2}\right) \otimes\left(T_{1} \oplus T_{2}\right)\right)\right) \\
& =2 S_{\min , \alpha}\left(T_{1} \oplus T_{2}\right)=S_{\min , \alpha}\left(T_{1}\right)+S_{\min , \alpha}\left(T_{2}\right) .
\end{aligned}
$$

On the other hand, if we first apply Prop. 1.1. and then weak additivity, we obtain that 19 and thus is upper bounded by $S_{\min , \alpha}\left(T_{1} \otimes T_{2}\right)$. The converse inequality is trivial.

\section{Consider}

$$
\begin{aligned}
\chi\left[\left(T_{1} \oplus T_{2}\right)^{\otimes 2}\right] & =2 \chi\left(T_{1} \oplus T_{2}\right)=2 \log \left[2^{\chi\left(T_{1}\right)}+2^{\chi\left(T_{2}\right)}\right] \\
= & \log \left[2^{2 \chi\left(T_{1}\right)}+2^{2 \chi\left(T_{2}\right)}+2^{\chi\left(T_{1}\right)+\chi\left(T_{2}\right)+1}\right] .
\end{aligned}
$$

This follows from first applying weak additivity and then the proposition 1.4. On the other hand, applying them in reverse order we have

$$
\begin{aligned}
& \chi\left[\left(T_{1} \oplus T_{2}\right)^{\otimes 2}\right] \\
& \quad=\log \left[2^{\chi\left(T_{1} \otimes T_{1}\right)}+2^{\chi\left(T_{2} \otimes T_{2}\right)}+2 \cdot 2^{\chi\left(T_{1} \otimes T_{2}\right)}\right] \\
& \quad=\log \left[2^{2 \chi\left(T_{1}\right)}+2^{2 \chi\left(T_{2}\right)}+2^{\chi\left(T_{1} \otimes T_{2}\right)+1}\right] .
\end{aligned}
$$

Together they prove the claimed equality.

For 3. we obtain by weak superadditivity,

$$
\begin{aligned}
H_{T_{1} \otimes T_{2}}(\rho) & =H_{\left(T_{1} \oplus T_{2}\right) \otimes\left(T_{1} \oplus T_{2}\right)}(0 \oplus \rho \oplus 0 \oplus 0) \\
& \geq H_{T_{1} \oplus T_{2}}\left(\rho_{1} \oplus 0\right)+H_{T_{1} \oplus T_{2}}\left(0 \oplus \rho_{2}\right) \\
& =H_{T_{1}}\left(\rho_{1}\right)+H_{T_{2}}\left(\rho_{2}\right) .
\end{aligned}
$$

Here, $\rho_{1}, \rho_{2}$ are reduced states of $\rho$. This proves 3 . and the statement 4 . follows in a similar way when replacing $\rho$ by a product state.

Proposition 3 (Unital channels) Proving one of the conjectures in proposition 2 for all pairs of identical unital channels would show the conjecture is true for arbitrary channels.

Proof. In [12] a unital channel $\tilde{T}$ is constructed for a given channel $T$ so that these two channels $\tilde{T}$ and $T$ share the following additivity properties: additivity of minimal output $\alpha$ Renyi entropy, and strong superadditivity and additivity of the convex closure of the output entropy. Hence these conjectures can be restricted to products $\tilde{T}_{1} \otimes \tilde{T}_{2}$ for all channels $T_{1}, T_{2}$. As for the HSW, we have the same reduction but for a different reason (See the remark below). Finally, for the above two unital channels $\tilde{T}_{1}, \tilde{T}_{2}$ we can construct the direct sum $\tilde{T}_{1} \oplus \tilde{T}_{2}$ which is again a unital channel. Then the result follows from the proof of proposition 2.

Remark: We explain local relation between minimal output entropy and the HSW capacity, which was implicitly written but not clear in [12]. Since the unital extension $\tilde{T}$ sort of mixes up outputs of $T$ we have the following formula.

$$
\chi\left(\tilde{T}_{1} \otimes \tilde{T}_{2}\right)=\log d_{1} d_{2}-S_{\min }\left(\tilde{T}_{1} \otimes \tilde{T}_{2}\right),
$$

where $d_{1}, d_{2}$ are the dimensions of the output spaces of $\tilde{T}_{1}$ and $\tilde{T}_{2}$ respectively. Hence the additivity of HSW capacity is equivalent to the additivity of the minimal output entropy for products of those extensions $\tilde{T}_{1} \otimes \tilde{T}_{2}$ by Eq. 22). Hence the additivity conjecture of the HSW capacity can also be restricted to products $\tilde{T}_{1} \otimes \tilde{T}_{2}$ for all channels $T_{1}, T_{2}$ by using global equivalence [8, 9, 10, 11].

Finally, we will discuss additivity issues for entanglement measures. The one already mentioned is the entanglement of formation which was introduced in [24]. Since then the following conjectures have been considered:

$$
\begin{aligned}
E_{F}(\rho) & \geq E_{F}\left(\rho_{1}\right)+E_{F}\left(\rho_{2}\right) \\
E_{F}\left(\rho_{1} \otimes \rho_{2}\right) & =E_{F}\left(\rho_{1}\right)+E_{F}\left(\rho_{2}\right) .
\end{aligned}
$$


In fact, both are again globally equivalent to the additivity of the HSW capacity and the minimal output entropy. Moreover, additivity would imply that $E_{F}$ equals an important operationally defined entanglement measure, the entanglement cost $E_{c}$, since $E_{c}(\rho)=\lim _{n \rightarrow \infty} E_{F}\left(\rho^{\otimes n}\right)$ [25].

The entanglement of formation $E_{F}(\rho)$ is the convex closure of output entropy $H_{T}(\rho)$ when $\mathrm{T}$ is a partial trace.

Following a similar strategy as above we will now show that strong additivity in the sense of Eq.24 is again implied by weak additivity (i.e., Eq. 24 with $\rho_{1}=\rho_{2}$ ). In fact, this will not only hold for $E_{F}$ but for any convex entanglement monotone [24, 26, 27]. The main reason behind is that every such functional satisfies [28]:

$$
f\left(\oplus_{i} \lambda_{i} \rho_{i}\right)=\sum_{i} \lambda_{i} f\left(\rho_{i}\right),
$$

where $\left\{\lambda_{i}\right\}$ is a probability distribution and $\rho_{i}$ are states as before.

\section{Proposition 4 (Convex entanglement monotones) [29]}

Suppose $f$ is a convex entanglement monotone which is weakly additive, i.e., $f\left(\rho_{1} \otimes \rho_{2}\right)=f\left(\rho_{1}\right)+f\left(\rho_{2}\right)$ for all $\rho_{1}=\rho_{2}$. Then $f$ is strongly additive in the sense that this holds also for all $\rho_{1} \neq \rho_{2}$.
Proof. Let $\rho=\frac{1}{2}\left(\rho_{1} \oplus \rho_{2}\right)$. Then

$$
f(\rho \otimes \rho)=2 f(\rho)=f\left(\rho_{1}\right)+f\left(\rho_{2}\right)
$$

Here, we applied the weak additivity and then (25). Applying them in reverse order we get

$$
\begin{aligned}
f(\rho \otimes \rho) & =\frac{1}{4}\left(\sum_{i, j=1}^{2} f\left(\rho_{i} \otimes \rho_{j}\right)\right) \\
& =\frac{1}{2}\left(f\left(\rho_{1}\right)+f\left(\rho_{2}\right)+f\left(\rho_{1} \otimes \rho_{2}\right)\right) .
\end{aligned}
$$

Using similar ideas, it has recently been shown that for regularized entanglement measures like $E_{c}$ or the asymptotic relative entropy of entanglement, monotonicity (i.e., essentially Eq. 25) and strong additivity are equivalent [18].

Acknowledgement M.F. would like to thank his supervisor Y.M.Suhov for constant encouragement and numerous discussions. M.W. thanks K.G. Vollbrecht for discussions and J.I. Cirac for support. Both authors thank M. B. Ruskai for bringing [30] to their attention.
[1] C. E. Shannon, “A Mathematical Theory of Communication", Bell System Technical Journal, 27 379E23 and 623E56 (1948).

[2] A. S. Holevo "The capacity of the quantum channel with general signal states", IEEE Trans. Info. Theory, 44, 269-273, (1998).

[3] B. Schumacher and M. D. Westmoreland, "Sending classical information via noisy quantum channels", Phys. Rev. A, 56, 131138, (1997).

[4] M.M. Wolf, J. Eisert, "Classical information capacity of a class of quantum channels ", New J. Phys. 7, 93 (2005); quant-ph/0412133

[5] C. King, "The capacity of the quantum depolarizing channel ", IEEE Trans. Inf. Theo. 49, 221 (2003); quant-ph/0204172

[6] C. King, "Additivity for unital qubit channels", J. Math. Phys. 43, 4641-4653, (2002).

[7] P.W. Shor, "Additivity of the Classical Capacity of Entanglement-Breaking Quantum Channels", J. Math. Phys. 43, 4334 (2002); quant-ph/0201149

[8] P.W. Shor, "Equivalence of Additivity Questions in Quantum Information Theory", Comm. Math. Phys., 246, Issue 3, 453472 (2004); quant-ph/0305035

[9] A.A. Pomeransky, "Strong superadditivity of the entanglement of formation follows from its additivity", Phys. Rev. A 68, 032317 (2003); quant-ph/0305056.

[10] K.M.R. Audenaert and S.L. Braunstein, "On Strong Subadditivity of the Entanglement of Formation", Comm. Math. Phys. 246 No 3, 443-452, (2004); quant-ph/0303045.

[11] K. Matsumoto, T. Shimono, and A. Winter, "Remarks on additivity of the Holevo channel capacity and of the entanglement of formation ", Commun. Math. Phys. 246, 437 (2004); quant-ph/0206148

[12] M. Fukuda, "Simplification of additivity conjecture in quantum information theory", quant-ph/0608010
[13] J.A. Smolin, F. Verstraete, A. Winter, "Entanglement of assistance and multipartite state distillation”, Phys. Rev. A 72, 052317 (2005); quant-ph/0505038

[14] M. Wolf, D. Perez-Garcia, "Quantum Capacities of Channels with small Environment ", Phys. Rev. A 75, 012303 (2007); quant-ph/0607070

[15] G. Smith, J.A. Smolin, A. Winter, "The quantum capacity with symmetric side channels ", quant-ph/0607039.

[16] P.W. Shor, J.A. Smolin, B.M. Terhal, "Nonadditivity of Bipartite Distillable Entanglement follows from Conjecture on Bound Entangled Werner States ”, Phys. Rev. Lett. 86, 2681 (2001); quant-ph/0010054

[17] K.G.H. Vollbrecht, R.F. Werner, M.M. Wolf, "On the irreversibility of entanglement distillation ", Phys. Rev. A 69, 062304 (2004); quant-ph/0301072

[18] F.G.S.L. Brandao, M. Horodecki, M.B. Plenio, S. Virmani, "Remarks on the equivalence of full additivity and monotonicity for the entanglement cost", quant-ph/0702136

[19] C. H. Bennett, P.W. Shor, J. A. Smolin, and A. V. Thapliyal,"Entanglement-assisted capacity of a quantum channel and the reverse Shannon theorem", IEEE Trans. Inf. Theory 48, 2637 (2002); quant-ph/0106052.

[20] P.W. Shor, "The quantum channel capacity and coherent information", lecture notes, MSRI Workshop on Quantum Computation (2002); I. Devetak, IEEE Trans. Inf. Th. 51, 44 (2005); S. Lloyd, Phys. Rev. A 55, 1613 (1997).

[21] M.B. Ruskai, "Inequalities for Quantum Entropy: A Review with Conditions for Equality ", J. Math. Phys. 43, 4358 (2002); erratum 46, 019901 (2005); quant-ph/0205064

[22] C. King and M. B. Ruskai, "Minimal entropy of states emerging from noisy quantum channels", IEEE Trans. Info. Theory, 47, 192-209 (2001); quant-ph/9911079

[23] R.F. Werner and A.S. Holevo, "Counterexample to an ad- 
ditivity conjecture for output purity of quantum channels", quant-ph/0203003

[24] C. H. Bennet, D. P. DiVincenzo, J. A. Smolin, W. K. Wootters, "Mixed-state entanglement and quantum error correction", Phys. Rev. A, 54, 3824-3851, (1996); quant-ph/9604024

[25] P.M. Hayden, M. Horodecki, B.M. Terhal, "The asymptotic entanglement cost of preparing a quantum state ", J. Phys. A: Math. Gen. 34, 6891 (2001); quant-ph/0008134

[26] G. Vidal, J. Mod. Opt., 47, 355 (2000); quant-ph/9807077

[27] V. Vedral and M. B. Plenio, Phys. Rev. A, 57, 1619 (1998); quant-ph/9707035

[28] M. Horodecki, "Simplifying monotonicity conditions for entanglement measures", Open Syst. Inf. Dyn., 12, 231 (2005), quant-ph/0412210

[29] This result is implicit in the recent work [18]. However, it has already been proven and been communicated by one of us at the ERATO conference, Kyoto 2003 (unpublished).

[30] E. Stormer, "A reduction theorem for capacity of positive maps", quant-ph/0510040 